Tôhoku Math. Journ.

24 (1972), 79-92.

\title{
ALMOST TANGENT MANIFOLDS OF SECOND ORDER
}

\author{
R. S. ClaAk aNd D. S. GoEl
}

(Received Oct. 20, 1971)

1. Let $M$ be a differentiable manifold of dimension $3 n$. A $G$-structure on $M$ whose group $G$ consists of all matrices of the form

$$
\left[\begin{array}{lll}
A & 0 & 0 \\
B & A & 0 \\
C & B & A
\end{array}\right]
$$

where $A \in G L\left(R^{n}\right)$, will be called an almost tangent structure of second order.

Suppose that such a structure is defined on $M$. Then $M$ is called an almost tangent manifold of second order. Denote by $P(M, G)$ the bundle of adapted frames. We define a $(1,1)$ tensor field $J$ on $M$ by specifying its components to be

$$
J_{0}=\left[\begin{array}{lll}
0 & 0 & 0 \\
I_{n} & 0 & 0 \\
0 & I_{n} & 0
\end{array}\right]
$$

relative to any adapted frame. Any local cross-section of $P(M, G)$ is an adapted moving frame of $M$. It can be identified with an independent set of vector fields $X_{1}, \cdots, X_{3 n}$ such that

$$
J X_{a}=X_{a+n}, \quad J X_{a+n}=X_{a+2 n}
$$

where $a=1, \cdots, n$.

The $(1,1)$ tensor field $J$ has constant rank $2 n$ and it satisfies the equation $J^{3}=0$. We shall show that any such tensor field determines an almost tangent structure of second order on $M$.

LEMmA 1.1. If a linear mapping $F: R^{3 n} \rightarrow R^{3 n}$ has rank $2 n$ and if $F^{3}=0$ then $\operatorname{Im} F=\operatorname{Ker} F^{2}$.

Proof. Since $\operatorname{Im} F \subset \operatorname{Ker} F^{2}$ it is sufficient to show that

$$
\operatorname{dim} \operatorname{Ker} F^{2}=\operatorname{dim} \operatorname{Im} F=2 n \text {. }
$$

Let $e_{2 n+1}, \cdots, e_{3 n}$ be a basis for $\operatorname{Ker} F$ and complete it to form a basis $e_{1}, \cdots, e_{3 n}$ for $R^{3 n}$. The vectors $F e_{1}, \cdots, F e_{2 n}$ belong to $\operatorname{Ker} F^{2}$ and they 
are linearly independent. Consequently the dimension of $\operatorname{Ker} F^{2}$ is at least $2 n$.

Suppose that $\operatorname{dim} \operatorname{Ker} F^{2}=2 n+s$. Since $\operatorname{Ker} F \subset \operatorname{Ker} F^{2}$ and since $\operatorname{dim} \operatorname{Ker} F=n$, there exist linearly independent vectors $f_{1}, \cdots, f_{n+s}$ which belong to $\operatorname{Ker} F^{2}$ and not to $\operatorname{Ker} F$. The vectors $F f_{1}, \cdots, F f_{n+s}$ belong to $\operatorname{Ker} F$ and they are linearly independent. Since Ker $F$ has dimension $n$, it follows that $s=0$.

Proposition 1.2. $M$ is a differentiable manifold of dimension $3 n$. Any $(1,1)$ tensor field $J$ of constant rank $2 n$ on $M$ and such that $J^{3}=0$ determines an almost tangent structure of second order on $M$.

Proof. Let $m$ be a given point of $M$ and choose a moving frame $\sigma$ whose domain $U$ contains $m$. The corresponding components of $J$ form a differentiable function $\mathscr{F}$ on $U$ whose matrix values have rank $2 n$.

Let $\mathscr{F}(m)=F$. The lemma shows that the kernel of the linear function $F^{2}: R^{3 n} \rightarrow R^{3 n}$ has dimension $2 n$. Let $e_{n+1}, \cdots, e_{3 n}$ be a basis for $\operatorname{Ker} F^{2}$ and complete it to form a basis $e_{1}, \cdots, e_{3 n}$ for $R^{3 n}$. The non-zero vectors

$$
F e_{1}, \cdots, F e_{n}, F^{2} e_{1}, \cdots, F^{2} e_{n}
$$

belong to $\operatorname{Ker} F^{2}$ and they are linearly independent. To prove this, suppose that

$$
\lambda^{a}\left(F e_{a}\right)+\mu^{a}\left(F^{2} e_{a}\right)=0 ;
$$

it follows that the vector

$$
\lambda^{a} e_{a}+\mu^{a}\left(F e_{a}\right)
$$

belongs to $\operatorname{Ker} F$ and hence to $\operatorname{Ker} F^{2}$ and so $\lambda^{a}=0(a=1, \cdots, n) ; \mu^{a} e_{a}$ will therefore belong to $\operatorname{Ker} F^{2}$ and so $\mu^{a}=0$. We conclude that the vectors

$$
e_{1}, \cdots, e_{n}, F e_{1}, \cdots, F e_{n}, F^{2} e_{1}, \cdots, F^{2} e_{n}
$$

are linearly independent.

Consequently the differentiable function

$$
\left[e_{1}, \cdots, e_{n}, \mathscr{F} e_{1}, \cdots, \mathscr{F} e_{n}, \mathscr{F}^{2} e_{1}, \cdots, \mathscr{F}^{2} e_{n}\right]
$$

on $U$ is non-singular at $m$. So it is non-singular on a neighbourhood $U^{\prime}$ of $m$. Denote this restriction by $T$. It now follows that

$$
\mathscr{F} T=\left[\mathscr{F} e_{1}, \cdots, \mathscr{F} e_{n}, \mathscr{F}^{2} e_{1}, \cdots, \mathscr{F}^{2} e_{n}, 0, \cdots, 0\right]=T J_{0}
$$

where we have defined the $3 n \times 3 n$ matrix $J_{0}$ in equation (1.1). Therefore, 
the tensor field $J$ has constant components $J_{0}$ relative to the moving frame $\sigma T$ on $U^{\prime}$.

We can construct a set of such moving frames whose domains cover $M$. On any intersection of their domains, two such frames differ by a matrix $L$ such that

$$
L J_{0}=J_{0} L \text {. }
$$

It is easy to deduce that $L$ has values in the almost tangent group $G$.

We use the tensor field $J$ to construct the Nijenhuis tensor $N$. This $(2,1)$ tensor field on $M$ is defined by

$$
\frac{1}{2} N(X, Y)=[J X, J Y]+J^{2}[X, Y]-J[X, J Y]-J[J X, Y]
$$

where $X, Y$ are two vector fields in $M$.

An almost tangent manifold $M$ of second order is said to be integrable if there exists a set of natural adapted frames whose domains cover $M$. It has been proved by J. Lehmann-Lejeune [1] that this is the case if and only if its Nijenhuis tensor is zero.

We give some examples of almost tangent manifolds of second order.

EXAMPLE 1.1. The tangent vectors of second order of any differentiable manifold $M$ form a manifold $T^{2} M$. Corresponding to any chart $x$ of $M$ we can define a standard chart $(x, y, z)$ of $T^{2} M$. Two such charts with intersecting domains are related by a change of co-ordinates which has Jacobian matrix

$$
\left[\begin{array}{ccc}
\frac{\partial \bar{x}^{a}}{\partial x^{b}} & 0 & 0 \\
\frac{\partial^{2} \bar{x}^{a}}{\partial x^{b} \partial x^{c}} y^{c} & \frac{\partial \bar{x}^{a}}{\partial x^{b}} & 0 \\
\frac{1}{2} \frac{\partial^{3} \bar{x}^{a}}{\partial x^{b} \partial x^{c} \partial x^{d}} y^{c} y^{d}+\frac{\partial^{2} \bar{x}^{a}}{\partial x^{b} \partial x^{c}} z^{c} & \frac{\partial^{2} \bar{x}^{a}}{\partial x^{b} \partial x^{c}} y^{c} & \frac{\partial \bar{x}^{a}}{\partial x^{b}}
\end{array}\right]
$$

The natural moving frames associated with these standard charts therefore define an integrable almost tangent structure of second order on $T^{2} M$.

EXAMPLE 1.2. We define a non-integrable almost tangent structure of second order on the sphere $S^{3} . S^{3}$ has a Lie group structure and its Lie algebra is isomorphic to the algebra of the purely imaginary quaternions (using standard multiplication). So there exists left-invariant vector fields $X_{1}, X_{2}, X_{3}$ on $S^{3}$ such that 


$$
\left[X_{1}, X_{2}\right]=X_{3},\left[X_{2}, X_{3}\right]=X_{1},\left[X_{3}, X_{1}\right]=X_{2} \text {. }
$$

We specify an almost tangent structure on $S^{3}$ by defining a (1.1) tensor field $J$ on $S^{3}$ by

$$
J X_{1}=X_{2}, J X_{2}=X_{3}, J X_{3}=0 \text {. }
$$

If this structure is integrable its Nijenhuis tensor $N$ must be zero. But

$$
\frac{1}{2} N\left(X_{2}, X_{3}\right)=X_{3} \neq 0 \text {. }
$$

We shall see in paragraph 5 that any manifold of dimension $3 n$ carrying an almost tangent structure of second order admits distributions of dimensions $n$ and $2 n$. It follows from results given by N. Steenrod ([2] p. 144) that no sphere of even dimension can carry such a structure. Neither can $S^{9}$ or $S^{21}$. After $S^{3}$, the first possibilities are $S^{15}$ and $S^{27}$.

EXAMPLE 1.3. Using a technique described by $\mathrm{S}$. Kobayashi and $\mathrm{K}$. Nomizu ([3], p. 138), we can use the structure on $S^{3}$ defined in the previous example to construct an almost tangent structure of second order on any orientable hypersurface $M$ in $R^{4}$. Let $g: M \rightarrow S^{3}$ be the spherical map of Gauss. The tangent spaces $T_{m} M$ and $T_{g m} S^{3}$ are parallel in $R^{4}$ and so they can be identified in a natural manner. From the (1.1) tensor field $J$ on $S^{3}$ we can therefore construct a similar tensor field on $M$.

2. A positive-definite Riemannian metric $S$ on an almost tangent manifold $M$ of second order determines an $O\left(R^{3 n}\right)$ structure on $M$. $S$ is said to be an almost tangent metric if the two structures on $M$ have a common subordinate structure.

We show how to construct such a metric starting from any given positive-definite Riemannian metric on $M$. This will show that such metrics exist when $M$ is paracompact.

LEMMA 2.1 $S$ is a given positive-definite Riemannian metric on an almost tangent manifold $M$ of second order and $m$ is any given point of $M$. There exists an adapted moving frame $\rho$ whose domain contains $m$ and relative to which $S$ has components of the form

$$
\left[\begin{array}{lll}
a & b & 0 \\
b^{\prime} & c & 0 \\
0 & 0 & I_{n}
\end{array}\right]
$$

where $b^{\prime}$ is the transpose of the $n \times n$ matrix $b$.

Proof. Choose any adapted moving frame $\sigma$ whose domain includes $m$ and relative to $\sigma$ let $S$ have components 


$$
\left[\begin{array}{ccc}
T & T_{1} & S_{1} \\
T_{1}^{\prime} & T_{2} & S_{2} \\
S_{1}^{\prime} & S_{2}^{\prime} & S_{3}
\end{array}\right]
$$

Because this matrix is positive-definite, we can choose a differentiable function $A$ (with non-singular matrix values) on a neighbourhood of $m$ such that

$$
A^{\prime} S_{3} A=I_{n} .
$$

Consider the adapted moving frame

$$
\rho=\sigma\left[\begin{array}{lll}
A & 0 & 0 \\
B & A & 0 \\
C & B & A
\end{array}\right]
$$

on a neighbourhood of $m$, where $B, C$ are defined by the equations

$$
S_{3} B+S_{2}^{\prime} A=0, S_{3} C+S_{2}^{\prime} B+S_{1}^{\prime} A=0 .
$$

It is easy to check that $S$ has components of the required form relative to $\rho$.

Proposition 2.2. A positive-definite Riemannian metric $S$ on an almost tangent manifold $M$ of second order is an almost tangent metric iff

$$
H J+J^{2} H^{2}=I
$$

where $H=S^{-1}(S J)^{\prime}$.

PROoF. If $S$ is an almost tangent metric there exists a set of adapted moving frames whose domains cover $M$ and relative to which $S$ has components $I_{3 n}$. It is therefore easy to verify that the above condition is necessary.

Suppose it is satisfied. Choose a special adapted moving frame $\rho$ whose domain contains a given point $m \in M$ and relative to which $S$ has components (2.1). Denote the inverse of this matrix by

$$
\left[\begin{array}{lll}
\alpha & \beta & 0 \\
\beta^{\prime} & \gamma & 0 \\
0 & 0 & I_{n}
\end{array}\right]
$$

The corresponding components of the tensor fields $H J$ and $J^{2} H^{2}$ reduce to

$$
\left[\begin{array}{lll}
\alpha c & \beta & 0 \\
\beta^{\prime} c & \gamma & 0 \\
0 & 0 & 0
\end{array}\right],\left[\begin{array}{lll}
0 & 0 & 0 \\
0 & 0 & 0 \\
0 & 0 & \alpha
\end{array}\right]
$$


and so the given condition shows that $\alpha=\gamma=I_{n}$ and $\beta=0 . \quad S$ therefore has components $I_{3 n}$ relative to the adapted moving frame $\rho$. We can construct a family of such moving frames whose domains cover $M$.

Proposition 2.3. A given positive-definite Riemannion metric $S$ on an almost tangent manifold $M$ of second order determines an almost tangent metric on $M$.

Proof. There exists a set of special adapted moving frames whose domains cover $M$ and which satisfy the conditions of Lemma 2.1. Any two such frames $\rho, \bar{\rho}$ with intersecting domains are related by an equation

$$
\bar{\rho}=\rho\left[\begin{array}{lll}
D & 0 & 0 \\
E & D & 0 \\
F & E & D
\end{array}\right]
$$

Since the components of $S$ relative to $\bar{\rho}$ are

$$
\left[\begin{array}{ccc}
D^{\prime} & E^{\prime} & F^{\prime} \\
0 & D^{\prime} & E^{\prime} \\
0 & 0 & D^{\prime}
\end{array}\right] \quad\left[\begin{array}{lll}
a & b & 0 \\
b^{\prime} & c & 0 \\
0 & 0 & I_{n}
\end{array}\right] \quad\left[\begin{array}{lll}
D & 0 & 0 \\
E & D & 0 \\
F & E & D
\end{array}\right]=\left[\begin{array}{ccc}
* & * & F^{\prime} D \\
* & * & E^{\prime} D \\
D^{\prime} F & D^{\prime} E & D^{\prime} D
\end{array}\right]
$$

it follows that $D \in O\left(R^{n}\right)$ and $E=F=0$. These special adapted moving frames therefore determine a $G \cap O\left(R^{3 n}\right)$ structure, and hence an almost tangent metric on $M$.

LEMMA 2.4. $S$ is a positive-definite Riemannian metric on an almost tangent manifold $M$ of second order. If its components relative to an adapted moving frame $\sigma$ are given in (2.2) then the corresponding components of the almost tangent metric constructed from $S$ are

$$
\left[\begin{array}{ccc}
S_{3}+S_{2} S_{3}^{-1} S_{2}^{\prime}+S_{1} S_{3}^{-1} S_{1}^{\prime} & S_{2}+S_{1} S_{3}^{-1} S_{2}^{\prime} & S_{1} \\
S_{2}^{\prime}+S_{2} S_{3}^{-1} S_{1}^{\prime} & S_{3}+S_{2} S_{3}^{-1} S_{2}^{\prime} & S_{2} \\
S_{1}^{\prime} & S_{2}^{\prime} & S_{3}
\end{array}\right]
$$

Proof: $\sigma$ can be expressed locally as

$$
\rho\left[\begin{array}{lll}
A & 0 & 0 \\
B & A & 0 \\
C & B & A
\end{array}\right]
$$

where $\rho$ is a special adapted moving frame relative to which $S$ has components (2.1). Then 


$$
\left[\begin{array}{ccc}
A^{\prime} & B^{\prime} & C^{\prime} \\
0 & A^{\prime} & B^{\prime} \\
0 & 0 & A^{\prime}
\end{array}\right] \quad\left[\begin{array}{lll}
a & b & 0 \\
b^{\prime} & c & 0 \\
0 & 0 & I_{n}
\end{array}\right] \quad\left[\begin{array}{ccc}
A & 0 & 0 \\
B & A & 0 \\
C & B & A
\end{array}\right]=\left[\begin{array}{ccc}
T & T_{1} & S_{1} \\
T_{1}^{\prime} & T_{2} & S_{2} \\
S_{1}^{\prime} & S_{2}^{\prime} & S_{3}
\end{array}\right]
$$

and consequently

$$
C^{\prime} A=S_{1}, B^{\prime} A=S_{2}, A^{\prime} A=S_{3} \text {. }
$$

Relative to $\rho$, the almost tangent metric has components $I_{3 n}$. Its components relative to $\sigma$ are therefore

$$
\left[\begin{array}{ccc}
A^{\prime} & B^{\prime} & C^{\prime} \\
0 & A^{\prime} & B^{\prime} \\
0 & 0 & A^{\prime}
\end{array}\right]\left[\begin{array}{lll}
A & 0 & 0 \\
B & A & 0 \\
C & B & A
\end{array}\right]
$$

and, by using equations (2.4), this reduces to the form stated above.

As a simple consequence of this lemma we have

Proposition 2.5. Two positive-definite Riemannian metrics $S, \bar{S}$ determine the same almost tangent metric on an almost tangent manifold iff $S J^{2}=\bar{S} J^{2}$.

If we start with an almost tangent metric $S$, then the metric constructed in proposition (2.3) is $S$ itself. Lemma 2.4 therefore leads to

Proposition 2.6. Relative to any adapted moving frame, any almost tangent metric has components of the form (2.3).

Suppose that $U$ is an open set of $M$ on which is defined an adapted moving frame $\sigma$ and that we are also given differentiable $n \times n$ matrix valued functions $S_{1}, S_{2}, S_{3}$ on $U$, the last of which is positive-definite. We can construct an almost tangent metric on $U$ by specifying its components relative to $\sigma$ to be of the form (2.2) where

$$
\begin{aligned}
T & =S_{3}+S_{2} S_{3}^{-1} S_{2}^{\prime}+S_{1} S_{3}^{-1} S_{1}^{\prime}, \\
T_{1} & =S_{2}+S_{1} S_{3}^{-1} S_{2}^{\prime}, \\
T_{2} & =S_{3}+S_{2} S_{3}^{-1} S_{2}^{\prime} .
\end{aligned}
$$

Suppose that an almost tangent metric is defined on an intersecting open set $\bar{U}$ using a moving frame $\bar{\sigma}$ and functions $\bar{S}_{1}, \bar{S}_{2}, \bar{S}_{3}$ and that on $U \cap \bar{U}$

$$
\sigma=\bar{\sigma}\left[\begin{array}{lll}
A & 0 & 0 \\
B & A & 0 \\
C & B & A
\end{array}\right]
$$


A straightforward calculation shows that the two metrics agree on $U \cap \bar{U}$ iff

$$
\begin{aligned}
& S_{1}=A^{\prime} \bar{S}_{1} A+B^{\prime} \bar{S}_{2} A+C^{\prime} \bar{S}_{3} A, \\
& S_{2}=A^{\prime} \bar{S}_{2} A+B^{\prime} \bar{S}_{3} A, \\
& S_{3}=A^{\prime} \bar{S}_{3} A .
\end{aligned}
$$

EXAMPLE 2.1. Starting from a positive-definite Riemannian metric on any given manifold $M$ we use the previous results to construct an almost tangent metric on the tangent manifold $T^{2} M$.

The moving frames associated with the standard charts $(x, y, z)$ of $T^{2} M$ are adapted for the almost tangent structure on $T^{2} M$. We can therefore construct an almost tangent metric on $T^{2} M$ by specifying functions

$$
S_{1}=\left[f_{a b}\right], S_{2}=\left[h_{a b}\right], S_{3}=\left[g_{a b}\right]
$$

on the domain $U$ of such chart $(x, y, z)$ provided that these are related to the corresponding functions on $\bar{U}$ by equations (2.5), where

$$
A=\left[\frac{\partial \bar{x}^{a}}{\partial x^{b}}\right], \quad B=\left[\frac{\partial^{2} \bar{x}^{a}}{\partial x^{b} \partial x^{c}} y^{c}\right], \quad C=\left[\frac{1}{2} \frac{\partial^{3} \bar{x}^{a}}{\partial x^{b} \partial x^{c} \partial x^{d}} y^{c} y^{d}+\frac{\partial^{2} \bar{x}^{a}}{\partial x^{b} \partial x^{c}} z^{c}\right] .
$$

We define the functions $g_{a b}$ to be the lifts into $T^{2} M$ of the components of the given metric on $M$ associated with the chart $x$. Then if $\Gamma_{b c}^{b}$ are the lifts of the corresponding Christoffel symbols we define

$$
\begin{gathered}
h_{a b}=g_{c b} \Gamma_{d a}^{c} y^{d}, \\
f_{a b}=\frac{1}{2}\left\{\frac{\partial}{\partial x^{c}}\left(g_{e b} \Gamma_{d a}^{b}\right)-g_{e f} \Gamma_{c a}^{e} \Gamma_{a b}^{f}\right\} y^{c} y^{d}+g_{d b} \Gamma_{c a}^{d} z^{c} .
\end{gathered}
$$

It is not difficult to show that equations (2.5) are satisfied.

3. Suppose that $M$ is an almost tangent manifold of second order. Apart from almost tangent metrics on $M$ (which are necessarily positivedefinite) we shall consider Riemannian metrics $S$ on $M$ such that

$$
S J=(S J)^{\prime} \text {. }
$$

We shall see that such a metric cannot be positive-definite but we shall suppose that it is non-singular. We call it a self-adjoint metric on $M$. That such metrics exist when $M$ is paracompact follows from

Proposition 3.1. If $S$ is any positive-definite Riemannian metric on an almost tangent manifold $M$ of second order, then the tensor field

$$
S J^{2}+\left(S J^{2}\right)^{\prime}+J^{\prime} S J-J^{\prime 2} S J^{2}
$$

is a self-adjoint metric on $M$. 
Proof. This $(2,0)$ tensor field is symmetric and it satisfies the equation (3.1). It is non-singular since if relative to any adapted moving frame $S$ has components given in (2.2), where $S_{3}$ is necessarily positivedefinite, then it has components

$$
\left[\begin{array}{ccc}
S_{1}+S_{1}^{\prime}+T_{2}-S_{3} & S_{2}+S_{2}^{\prime} & S_{3} \\
S_{2}+S_{2}^{\prime} & S_{3} & 0 \\
S_{3} & 0 & 0
\end{array}\right] .
$$

EXAMPLE 3.1. Starting with the almost tangent metric on $T^{2} M$ described in example 2.1 leads to a self-adjoint metric on $T^{2} M$. Its components associated with a standard chart $(x, y, z)$ are of the form (3.2), where $S_{1}, S_{2}, S_{3}$ are defined in example 2.1 and $T_{2}=S_{3}+S_{2} S_{3}^{-1} S_{2}^{\prime}$. It is easy to verify that this is the lift into $T^{2} M$ of the original Riemannian metric on $M$ as defined by $\mathrm{K}$. Yano and S. Ishihara [4].

Proposition 3.2. Relative to any adapted moving frame, any selfadjoint metric has components

$$
\left[\begin{array}{lll}
T & T_{1} & T_{2} \\
T_{1} & T_{2} & 0 \\
T_{2} & 0 & 0
\end{array}\right]
$$

where $T, T_{1}, T_{2}$ are symmetric $n \times n$ matrices and $\operatorname{det} T_{2} \neq 0$.

Proof. Suppose that the metric has components of the form (2.2) where $T, T_{2}, S_{3}$ are symmetric. The condition (3.1) shows that

$$
\left[\begin{array}{ccc}
T_{1} & S_{1} & 0 \\
T_{2} & S_{2} & 0 \\
S_{2}^{\prime} & S_{3} & 0
\end{array}\right]=\left[\begin{array}{ccc}
T_{1}^{\prime} & T_{2}^{\prime} & S_{2} \\
S_{1}^{\prime} & S_{2}^{\prime} & S_{3}^{\prime} \\
0 & 0 & 0
\end{array}\right]
$$

and this leads to the result stated.

Lemma 3.3. $S$ is a given self-adjoint metric on an almost tangent manifold $M$ of second order and $m$ is any given point of $M$. There exists an adapted moving frame $\rho$, whose domain contains $m$, relative to which $S$ has constant components of the form

$$
\left[\begin{array}{ccc}
0 & 0 & K \\
0 & K & 0 \\
K & 0 & 0
\end{array}\right]
$$

where $K$ is some diagonal $n \times n$ matrix of the form

$$
\operatorname{diag}\{1,1, \cdots-1, \cdots\} \text {. }
$$


Proof. Choose any adapted moving frame $\sigma$ whose domain includes $m$ and, relative to $\sigma$, let $S$ have components (3.3). The differentiable function $T_{2}$ is symmetric and non-singular. Consequently we can find a differentiable function $A$ (with values in $G L\left(R^{n}\right)$ ) on some neighbourhood $U$ of $m$ such that

$$
A^{\prime} T_{2} A=K
$$

on $U$, where $K$ is some fixed matrix diag $\{1,1, \cdots,-1, \cdots\}$. Then using the differentiable functions $B, C$ defined by

$$
T_{1} A+2 T_{2} B=0,2 T A+3 T_{1} B+4 T_{2} C=0
$$

we define the adapted moving frame

$$
\rho=\sigma\left[\begin{array}{lll}
A & 0 & 0 \\
B & A & 0 \\
C & B & A
\end{array}\right]
$$

on a neighbourhood of $m$. This has the property required.

From this lemma we deduce

Proposition 3.4. Every self-adjoint metric $S$ on a connected almost tangent manifold of second order and dimension $3 n$ has constant signature $(n+s, 2 n-s)$, where $0 \leq s \leq n$.

Proof. Since any Riemannian metric on a connected manifold has constant signature, we need only calculate the signature of $S$ at just one point. Suppose that $S$ has components of the form (3.4). If the diagonal matrix $K$ has signature $(s, n-s)$ then this matrix, which is congruent to

$$
\left[\begin{array}{ccc}
K & 0 & 0 \\
0 & K & 0 \\
0 & 0 & -K
\end{array}\right]
$$

has signature $(n+s, 2 n-s)$.

4. Suppose that $M$ is an almost tangent manifold of second order. A connection on the frame bundle $H\left(M, G L\left(R^{3 n}\right)\right.$ ) is called an almost tangent connection if every horizontal vector at each point $p \in P$ is tangent to the submanifold $P$ of $H$. Such a linear connection determines a connection on the adapted frame bundle $P(M, G)$. Conversely any connection on $P(M, G)$ arises in this way from a unique almost tangent connection. 
Suppose that $\nabla$ is any linear connection on $M$. Corresponding to any moving frame $\sigma=X_{1}, \cdots, X_{3 n}$ in $M$ with domain $U$, the connection $\nabla$ has coefficients $\Gamma_{j k}^{i}$ defined on $U$ by

$$
\nabla_{X_{i}} X_{j}=\Gamma_{i j}^{h} X_{h} \text {. }
$$

The corresponding local connection form

$$
\omega=\left[\Gamma_{h j}^{i} \theta^{h}\right]
$$

on $U$ has values in the Lie algebra of $G L\left(R^{3 n}\right) . \quad \nabla$ is an almost tangent connection iff such local forms $\omega$ have values in the Lie algebra of $G$ whenever $\sigma$ is an adapted moving frame. We deduce

Proposition 4.1. A linear connection on an almost tangent manifold $M$ of second order is an almost tangent connection iff its coefficients corresponding to each adapted moving frame satisfy the conditions

$$
\begin{aligned}
\Gamma_{h c}^{a} & =\Gamma_{h+n}^{a+n}=\Gamma_{h+2 n}^{a+2 n}, \Gamma_{h c}^{a+n}=\Gamma_{h c+n}^{a+2 n}, \\
\Gamma_{h c+n}^{a} & =\Gamma_{h c+2 n}^{a}=\Gamma_{h c+2 n}^{a+n}=0,
\end{aligned}
$$

for all $h=1, \cdots, 3 n ; a, c=1, \cdots, n$.

It is sufficient to verify this condition for a set of adapted moving frames whose domains cover $M$ and so we have

EXAMPLE 4.1. Starting with a linear connection $\nabla$ for any given manifold $M$ whose coefficients associated with a chart $x$ are $\Gamma_{b c}^{a}$, K. Yano and S. Ishihara [4] have defined a connection for $T^{2} M$ whose coefficients $\bar{\Gamma}^{i}{ }_{j k}$ associated with a standard chart $(x, y, z)$ are all zero except

$$
\begin{aligned}
\bar{\Gamma}_{b c}^{a} & =\bar{\Gamma}_{b c+n}^{a+n}=\bar{\Gamma}_{b+n c}^{a+n}=\bar{\Gamma}_{b c+2 n}^{a+2 n}=\bar{\Gamma}_{b+n c+n}^{a+2 n}=\bar{\Gamma}_{b+2 n c}^{a+2 n}=\Gamma_{b c}^{a}, \\
\bar{\Gamma}_{b c}^{a+n} & =\bar{\Gamma}_{b c+n}^{a+2 n}=\bar{\Gamma}_{b+n c}^{a+2 n}=y^{d} \frac{\partial}{\partial x^{d}} \Gamma_{b c}^{a}, \\
\bar{\Gamma}_{b c}^{a+2 n} & =z^{d} \frac{\partial}{\partial x^{d}} \Gamma_{b c}^{a}+\frac{1}{2} y^{d} y^{e} \frac{\partial^{2}}{\partial x^{d} \partial x^{e}} \Gamma_{b c}^{a},
\end{aligned}
$$

for all $a, \cdots, e=1, \cdots, n$. This connection is therefore an almost tangent connection for $T^{2} M$.

An alternative condition that a linear connection on $M$ is an almost tangent connection was given in [5]:

Proposition 4.2. A linear connection $\nabla$ on an almost tangent manifold $M$ is an almost tangent connection iff

$$
\nabla_{X} J=0
$$


for all vector fields $X$ in $M$.

This is equivalent to the condition

$$
\nabla_{X}(J Y)-J\left(\nabla_{X} Y\right)=0
$$

for all vector fields $X, Y$ in $M$.

5. Suppose that $M$ is an almost tangent manifold of second order. Consider the group $G^{+}$consisting of the matrices

$$
\left[\begin{array}{lll}
A & 0 & 0 \\
D & B & 0 \\
E & F & C
\end{array}\right]
$$

where $A, B, C \in G L\left(R^{n}\right)$. Since $G^{+}$contains the almost tangent group $G$ as a subgroup, it follows that $M$ carries a $G^{+}$-structure with respect to which its almost tangent structure is subordinate.

We shall study $G^{+}$-connections on $M$. Any adapted moving frame of $M$ is also adapted for the $G^{+}$-structure. Consequently a linear connection on $M$ is a $G^{+}$-connection iff the local connection forms associated with the adapted moving frames of $M$ have values in the Lie algebra of $G^{+}$. This leads to

Proposition 5.1. A linear connection on an almost tangent manifold $M$ of second order is a $G^{+}$-connection iff its coefficients corresponding to each adapted moving frame of $M$ satisfy the conditions

$$
\Gamma_{h c+n}^{a}=\Gamma_{h c+2 n}^{a}=\Gamma_{h c+2 n}^{a+n}=0
$$

for all $h=1, \cdots, 3 n ; a, c=1, \cdots, n$.

An analogue of proposition 4.2 is given in

Proposition 5.2. A linear connection $\nabla$ on an almost tangent manifold $M$ of second order is a $G^{+}$-connection iff

$$
J\left(\nabla_{X} J^{2}\right)=0, J^{2}\left(\nabla_{X} J\right)=0
$$

for all vector fields $X$ in $M$.

Proof. Proposition (5.1) implies that $\nabla$ is a $G^{+}$-connection iff

$$
J\left(\nabla_{X_{i}}\left(J^{2} X_{j}\right)\right)=0, J^{2}\left(\nabla_{X_{i}}\left(J X_{j}\right)\right)=0
$$

(for all $i, j=1, \cdots, 3 n$ ) whenever $X_{1}, \cdots, X_{3 n}$ is an adapted moving frame in $M$. Condition (5.1) is equivalent to the condition

$$
J\left(\nabla_{X}\left(J^{2} Y\right)\right)=0, J^{2}\left(\nabla_{X}(J Y)\right)=0
$$

for all vector fields $X, Y$ in $M$. Clearly if condition (5.3) is satisfied 
then so is condition (5.2). Using the fact that $J^{3}=0$, it is easy to prove that the converse is true also.

The (1.1) tensor field $J$ determines a linear mapping

$$
J_{m}: v \longrightarrow J(m) v
$$

on each tangent vector space $T_{m} M$. The function

$$
\text { Ker } J: m \longrightarrow \text { kernel } J_{m}
$$

is an $n$-dimensional distribution on $M$. If vector fields $X_{1}, \cdots, X_{3 n}$ form an adapted moving frame in $M$, then $X_{2 n+1}, \cdots, X_{3 n}$ form a local basis for Ker J. It therefore defines a structure on $M$ whose group $G_{1}$ consists of the non-singular matrices

$$
\left[\begin{array}{ccc}
A & H & 0 \\
D & B & 0 \\
E & F & C
\end{array}\right]
$$

with respect to which the $G^{+}$-structure on $M$ is subordinate.

A similar argument shows that the $2 n$-dimensional distribution $\mathrm{Ker} \mathrm{J}^{2}$ determines a structure on $M$ whose group $G_{2}$ consists of the non-singular matrices

$$
\left[\begin{array}{lll}
A & 0 & 0 \\
D & B & H \\
E & F & C
\end{array}\right]
$$

with respect to which the $G^{+}$-structure on $M$ is also subordinate.

A linear connection on $M$ is a $G^{+}$-connection iff it is a connection for both the $G_{1}$ and $G_{2}$ structures. This leads to

Proposition 5.3. $\nabla$ is a linear connection on an almost tangent manifold $M . v(0)$ is any vector at a point $m(0)$ of $M$ and $v(t)$ are the vectors obtained by parallel displacement along a curve $m(t)$. If $\nabla$ is a $G^{+}$-connection, then the vectors $v(t)$ belong to Ker $J$ if $v(0)$ belongs to Ker $J$ and they belong to $\mathrm{Ker} J^{2}$ if $v(0)$ belongs to $\mathrm{Ker} J^{2}$.

As another consequence of the previous remark we have

EXAMPLE 5.1. Consider the sphere $S^{3}$ with its almost tangent structure as defined in example 1.2. In this case the distribution $\operatorname{Ker} J^{2}$ admits a global basis $X_{2}, X_{3}$ and it is not integrable since $\left[X_{2}, X_{3}\right]=X_{1}$ which is not tangent to the distribution $\operatorname{Ker} J^{2}$. Therefore the $G_{2^{-}}$ structure on $S^{3}$ is not integrable and does not admit a torsion-free connection. It follows that $S^{3}$ cannot carry a torsion-free $G^{+}$-connection. 
Consequently the $G^{+}$-structure on $S^{3}$ is not integrable.

\section{REFERENCES}

[1] J. Lehmann-Lejeune, Sur 1'intégrabilité de certaines G-structures, C. R. Acad. Sci. Paris, 258 (1964), 5326-5329.

[2] N. Steenrod, Topology of fibre bundles, Princeton University Press 1951.

[3] S. Kobayashi aND K. Nomizu, Foundations of differential geometry Vol. II, Interscience Publishers 1969.

[4] K. Yano AND S. Ishihara, Differential geometry of tangent bundles of order 2, Kōdai Math. Sem. Rep., 20 (1968), 318-354.

[5] R. S. Clark aNd M. BRUCKHeimer, Tensor structures on a differentiable manifold, Annali di matematica, 54 (1961), 123-142.

DepartMent OF Mathematics

The UNIVERSity OF CALGary

Calgary, Alberta,

Canada 